\begin{tabular}{|c|l|}
\hline Title & Negative viscosity of a liquid crystal in the presence of turbulence \\
\hline Author(s) & $\begin{array}{l}\text { Orihara, Hiroshi; Harada, Yuko; Kobay ashi, Fumiaki; Sasaki, Yuji; Fujii, Shuji; Satou, Y uki; Goto, Y oshitomo; } \\
\text { Nagaya, Tomoyuki }\end{array}$ \\
\hline Citation & $\begin{array}{l}\text { Physical Review E, 99(1),012701 } \\
\text { https://doi.org/10.1103/PhysRevE.99.012701 }\end{array}$ \\
\hline Issue Date & 2019-01-02 \\
\hline Doc URL & http://hdl.handle.net/2115/73006 \\
\hline Rights & @2019 A merican Physical Society \\
\hline Type & article \\
\hline File Information & PhysRevE.99.012701.pdf \\
\hline
\end{tabular}

Instructions for use 


\title{
Negative viscosity of a liquid crystal in the presence of turbulence
}

\author{
Hiroshi Orihara, ${ }^{1, *}$ Yuko Harada, ${ }^{1}$ Fumiaki Kobayashi, ${ }^{1}$ Yuji Sasaki, ${ }^{1}$ Shuji Fujii, ${ }^{1}$ Yuki Satou, ${ }^{2}$ \\ Yoshitomo Goto, ${ }^{3,4}$ and Tomoyuki Nagaya ${ }^{2,4,5, \dagger}$ \\ ${ }^{1}$ Division of Applied Physics, Hokkaido University, Sapporo 060-8628, Japan \\ ${ }^{2}$ Division of Electrical and Electronic Engineering, Oita University, Oita 870-1192, Japan \\ ${ }^{3}$ Beppu University Junior College, Beppu 874-8501, Japan \\ ${ }^{4}$ Division of Materials Science and Production Engineering, Oita University, Oita 870-1192, Japan \\ ${ }^{5}$ Division of Natural Sciences, Oita University, Oita 870-1192, Japan
}

(Received 5 August 2018; published 2 January 2019)

\begin{abstract}
We report on the discovery of enormous negative viscosity in a nematic liquid crystal in the presence of turbulence induced by electric fields. As the negative viscosity in this system is so large, we are able to observe several phenomena originating from it. For example, we observe a spontaneous shear flow that rotates the upper disk of a rheometer, as well as the reversal of the rotational direction upon applying an external torque in the opposite direction. Hysteresis loops are also observed in the shear-stress-shear-rate curves, which is reminiscent of those seen for ferromagnetic and ferroelectric materials. The similarities between the phenomena observed for our system and ferroic materials are comprehensively demonstrated, although the two systems are fundamentally different in that the former is out of equilibrium. We elucidate the origin of the negative viscosity and propose a simple model that reproduces the phenomena observed in this active fluid.
\end{abstract}

DOI: 10.1103/PhysRevE.99.012701

\section{INTRODUCTION}

Numerous experimental [1-5] and theoretical [6-14] studies have been conducted in an attempt to observe negative viscosity. The viscosity of a fluid is a measure of its resistance to flow. In the case of negative viscosity, the flow is amplified by negative resistance even in the absence of external stress, resulting in spontaneous flow. In magnetic fluids (MFs) [6,7], which are colloidal solutions of magnetic particles, the viscosity has been observed to decrease upon application of an alternating magnetic field, although it remains positive [1]. Each particle is rotated by the alternating magnetic field, which generates a vortex flow around the particle. Other systems include active suspensions of bacteria [2-5,8-14]. Recently, López et al. observed negative viscosity in suspensions of Escherichia coli, in which the bacteria generated a specific flow field that reduced the apparent viscosity by organizing themselves [2]. Because the viscosity was very low (approximately $-10^{-1} \mathrm{mPa}$ ), the authors used a rheometer specially designed for investigating low-viscosity fluids.

Recently, Nagaya et al. demonstrated a remarkable reduction of viscosity in a nematic liquid crystal (NLC) $[15,16]$. Typical NLCs consist of rodlike molecules with longrange orientational order, where the average direction of the molecules is designated by a unit vector $\mathbf{n}$ that is referred to as the director $[17,18]$. One of the most remarkable properties of NLCs is the coupling between the director and the flow; a change in the director can induce flow and vice versa [17-19]. Furthermore, the director can be controlled by external

\footnotetext{
*orighara@eng.hokudai.ac.jp

†nagaya@oita-u.ac.jp
}

electric fields owing to the dielectric anisotropy $\Delta \varepsilon=\varepsilon_{\|}-\varepsilon_{\perp}$, where $\varepsilon_{\|}$and $\varepsilon_{\perp}$ are the relative dielectric constants parallel and perpendicular to $\mathbf{n}$, respectively. The director tends to be parallel or perpendicular to the electric field for $\Delta \varepsilon>0$ and $\Delta \varepsilon<0$, respectively, which reduces the interaction energy with the electric field. The interaction energy density can be expressed as $f_{\mathrm{el}}=-\frac{1}{2} \varepsilon_{0} \varepsilon_{\perp} E^{2}-\frac{1}{2} \varepsilon_{0} \Delta \varepsilon(\mathbf{n} \cdot \mathbf{E})^{2}$, where $\varepsilon_{0}$ is the dielectric constant in a vacuum and $\mathbf{E}$ is the applied electric field $[17,18]$. This relationship implies that the torque exerted on the director is proportional to the square of the electric field. The applied electric field causes orientational change of the director and then a change in viscosity. Such fluids whose viscosity can be controlled by electric fields are referred to as electrorheological (ER) fluids [20]. Although typical ER fluids consist of suspensions of dielectric particles in an electrically insulating oil, some studies have also investigated the ER properties of NLCs [21-23]. In NLCs, the electrorheological effect is dependent on the dielectric anisotropy, as was clearly demonstrated by Nagaya $e t$ al. in experiments using a series of mixtures of two NLCs with positive and negative dielectric anisotropies [16]. These authors prepared mixtures containing different fractions of the two NLCs with dielectric anisotropies $\Delta \varepsilon$ ranging from -0.483 to 0.245 . In the low-voltage region, the viscosity monotonically increased for all of the mixtures, while in the high-voltage region, the viscosity of the mixtures with negative dielectric anisotropy reached a maximum and then decreased, whereas that of the mixtures with positive dielectric anisotropy continued to increase. However, even in the mixture with the lowest dielectric anisotropy of -0.483 , the viscosity did not reach a negative value. It should be noted that in the high-voltage region, electroconvection (EC) was observed in all of the mixtures. In general, NLCs exhibit EC under high electric fields, which 


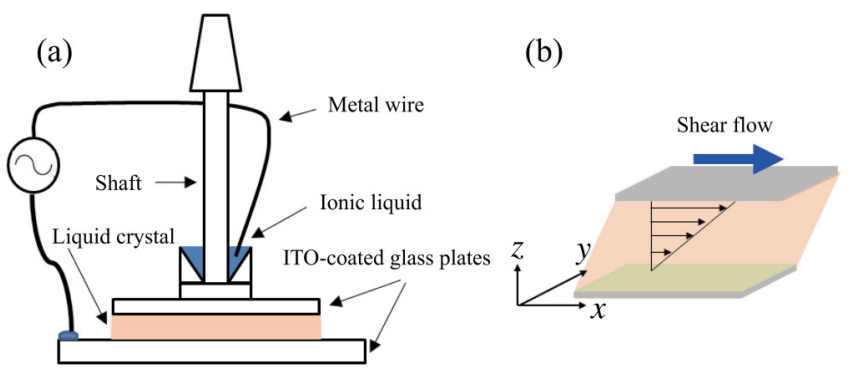

FIG. 1. Schematic illustration of experimental setup. (a) The upper disk is composed of glass completely coated with ITO and attached to the metal sensor of a rheometer. The lower stage is also composed of glass with the top surface coated with ITO. Microscopic observations can be made through the lower glass stage. (b) The $x$, $y$, and $z$ axes are taken along the flow, vorticity, and velocity gradient (electric field) directions, respectively.

is caused by the conduction of impurity ions and whose appearance depends on both the dielectric and conductive anisotropies [17,18,24-29]. Although EC occurs under both $\mathrm{dc}$ and ac voltages, an ac voltage is generally used to avoid degradation due to ion injection at the electrodes [18]. The convective pattern changes from regular rolls to turbulence through various patterns upon changing the amplitude and frequency of the ac electric field [26].

In this paper we demonstrate that the viscosity of an NLC with negative dielectric anisotropy becomes negative at high electric fields, where the absolute value of the negative viscosity is several hundred times greater than that of the previously reported bacterial suspensions, and report on some phenomena related to the negative viscosity such as the occurrence of hysteresis loops. This paper is organized as follows. In Sec. II we describe the experimental setup and sample preparation. In Sec. III we present the experimental results, including the observation of negative viscosity, hysteresis loops, and S-shaped curves, temperature and frequency dependences, and a self-oscillation due to the negative viscosity. In Sec. IV we discuss the mechanism underlying the negative viscosity based on the Ericksen-Leslie theory and propose a simple model that reproduces the experimental results. In Sec. V we summarize our findings and offer some concluding remarks.

\section{EXPERIMENTAL DETAILS}

Rheological measurements and microscopic observations were performed on the NLC $p$-methoxybenzylidene- $p^{\prime}-n$ butylaniline (MBBA), which exhibits negative dielectric anisotropy $\left(\Delta \varepsilon=-0.631\right.$ at $25^{\circ} \mathrm{C}$ ) and is a typical NLC that has frequently been used to study EC. We used a commercially available sample of MBBA (Tokyo Chemical Industry) as is, without dopant ions; the measured conductivity at $25^{\circ} \mathrm{C}$ was $0.9 \times 10^{-7} \Omega^{-1} \mathrm{~m}^{-1}$, which is sufficiently large to induce EC [18]. As shown in Fig. 1(a), the liquid crystal sample was sandwiched between the upper disk (50 mm diameter) and the lower stage of a rheometer (MCR-302, Anton Paar), both of which were fabricated from glass coated with indium tin oxide (ITO). The upper disk and lower stage were mutually parallel and separated by a gap of $100 \mu \mathrm{m}$. Since the shear rate depends on the measurement position, for the purposes of this
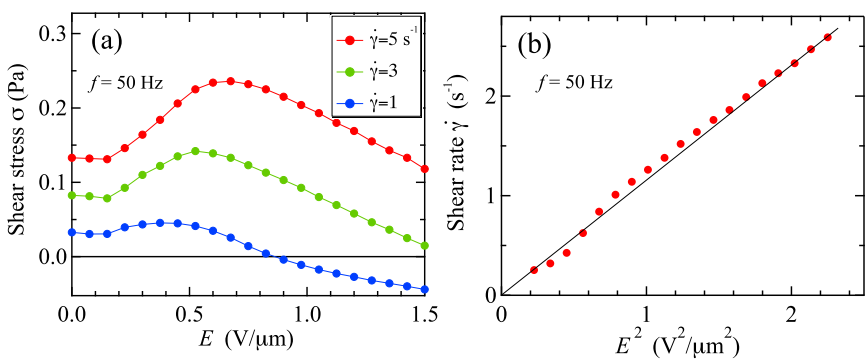

FIG. 2. Negative shear stress and spontaneous shear flow. (a) Electric field dependences of shear stress at three different shear rates of 1,3 , and $5 \mathrm{~s}^{-1}$. For the measurements, ac electric fields with a frequency of $50 \mathrm{~Hz}$ were applied and the amplitude is used to express the electric field strength. At the lowest shear rate, the shear stress becomes negative at high electric fields. (b) Dependence of spontaneous shear rate (which is proportional to the angular velocity of the upper disk) on the square of the electric field. The measurements were performed at zero shear stress.

study it was defined as that at the periphery of the upper disk. The shear stress at the edge of the disk was calculated from the measured torque by assuming that the fluid is Newtonian. No surface treatment was performed for liquid crystal alignment. A voltage was applied between the upper disk and the lower stage through a metal wire and an ionic liquid [1-ethyl-3methylimidazolium bis(trifluoromethanesulfonyl)imide] filling a tray on the upper disk to avoid friction between the wire and upper disk. Microscopic observations were made through the glass disk and stage using a microscope (IX73, Olympus) and a video camera (ORCA-Flash4.0, Hamamatsu), respectively.

\section{RESULTS}

\section{A. Negative shear stress and spontaneous shear flow}

Measurements were performed at $25^{\circ} \mathrm{C}$ in the nematic phase. Figure 2(a) shows the voltage dependence of the shear stress measured at constant shear rates of 1,3 , and $5 \mathrm{~s}^{-1}$, where the frequency of the applied ac electric field was $50 \mathrm{~Hz}$ and $E$ represents the amplitude. For each shear rate, the shear stress first increased to a maximum and then decreased monotonically with increasing applied electric field. In particular, for a shear rate of $1 \mathrm{~s}^{-1}$, the shear stress reached zero at approximately $0.85 \mathrm{~V} / \mu \mathrm{m}$ and thereafter became negative. The viscosity at $1.5 \mathrm{~V} / \mu \mathrm{m}$ was $-44 \mathrm{mPa} \mathrm{s}$, the absolute value of which is considerably greater than that of $-0.1 \mathrm{mPa}$ s reported for the aforementioned bacterial suspension. The negative shear stress creates spontaneous shear flow, resulting in the rotation of the upper disk of the rheometer if it is allowed to freely rotate, that is, at a constant shear stress of $0 \mathrm{~Pa}$. We successfully observed this spontaneous rotation, as well as the reversal of the rotation direction upon the application of an external force (see video 1 in [30] and the Appendix, Sec. 1). Figure 2(b) shows the dependence of the spontaneous shear rate (which is proportional to the angular velocity of the upper disk) on the square of the applied electric field. It can be seen that the shear rate was nearly proportional to the square of the electric field. This square dependence may stem from the 

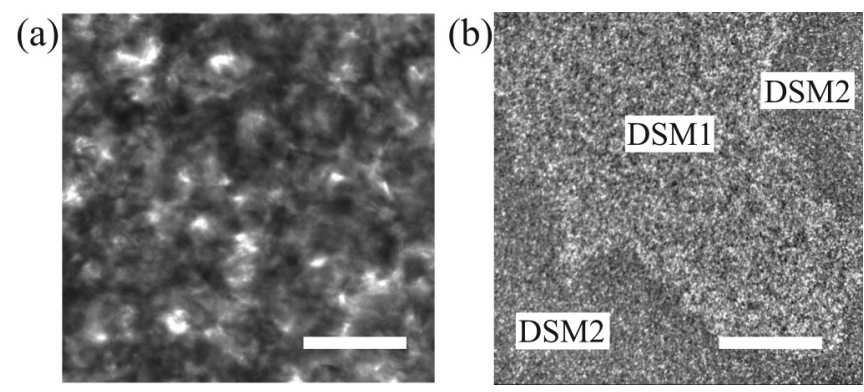

FIG. 3. (a) Turbulence observed at $E=1.5 \mathrm{~V} / \mu \mathrm{m}$ and $f=$ $50 \mathrm{~Hz}$, which corresponds to the DSM2 state (see video 2 in [30]). The scale bar is $50 \mu \mathrm{m}$. Note that a macroscopic flow can be observed from left to right in the video. (b) Coexistence of the DSM1 and DSM2 states. The image was clipped from a movie taken after applying an electric field of $E=0.3 \mathrm{~V} / \mu \mathrm{m}$ and $f=50 \mathrm{~Hz}$ (see video 3 in [30]). The bright and dark domains correspond to the DSM1 and DSM2 states, respectively. The scale bar is $1 \mathrm{~mm}$.

square dependence of the director torque on the electric field as described above.

As MBBA is somewhat susceptible to hydrolysis, which may be accelerated by ionic conduction in the presence of an electric field, we investigated the possibility of sample degradation. The conductivity of our sample at $25^{\circ} \mathrm{C}$ was $0.9 \times 10^{-7} \Omega^{-1} \mathrm{~m}^{-1}$. In our sample with a volume of $\pi r^{2} h \approx$ $200 \mu \mathrm{L}$ (with $r=25 \mathrm{~mm}$ the radius of the upper plate and $h=100 \mu \mathrm{m}$ the gap between the upper disk and the lower stage), the total current (amplitude) and power were $0.27 \mathrm{~mA}$ and $20 \mathrm{~mW}$, respectively, at an applied voltage of $150 \mathrm{~V}$ (amplitude) and a frequency of $50 \mathrm{~Hz}$. We checked for the occurrence of degradation under these conditions by monitoring the time dependence of the spontaneous shear rate over $10 \mathrm{~h}$. The decrease in the spontaneous shear rate was approximately $1 \%$. As the longest measurement made in the present study was $7 \mathrm{~h}$, sample degradation can therefore be neglected.

\section{B. Turbulence caused by electric fields}

When a liquid crystal exhibits the negative shear stress and spontaneous shear flow discussed above, turbulence occurs [24-29]. In general, NLCs reach turbulent states through a series of bifurcations [26]. Two types of turbulence are known, which are referred to as dynamic scattering modes (DSMs) 1 and 2 [25]. The former appears at lower electric fields than the latter. It was shown that the transition between the two DSM states occurs via nucleation of DSM2 and its subsequent growth in an increasing electric field, and disclinations are created in the DSM2 state during this transition [25].

Figure 3(a) and video 2 in [30] show the typical turbulence observed at $E=1.5 \mathrm{~V} / \mu \mathrm{m}$ and $f=50 \mathrm{~Hz}$. The turbulence was confirmed to be DSM2 by observing the coexistence of the DSM1 and DSM2 states and the growth of the DSM2 domains upon applying an electric field of $E=0.3 \mathrm{~V} / \mu \mathrm{m}$ [Fig. 3(b) and video 3 in [30]]. Since disclinations scatter the incident light, the DSM2 domains are darker. The transition electric field was determined to be $E_{\mathrm{c}}=0.16 \mathrm{~V} / \mu \mathrm{m}$ by slowly varying the applied electric field around $E_{\mathrm{c}}$. This obtained value of $E_{\mathrm{c}}$ is smaller than typical values reported to date, because the sample gap $(100 \mu \mathrm{m})$ was considerably
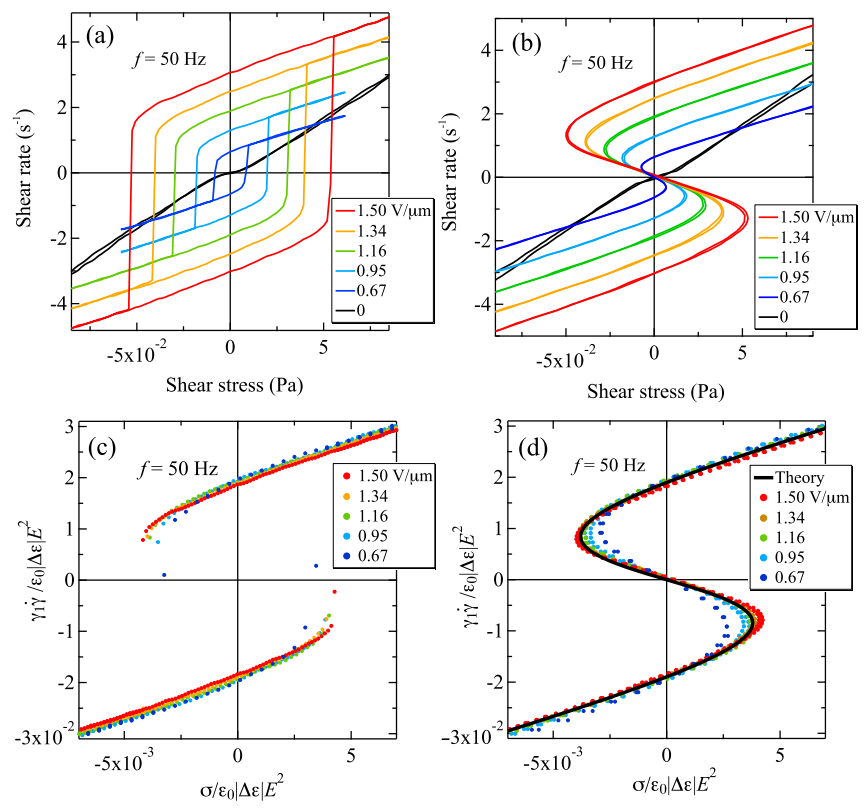

FIG. 4. Hysteresis loops, S-shaped curves, and scaling relation. (a) and (b) Relationships between shear stress and shear rate at various fields under (a) controlled shear stress and (b) controlled shear rate. Hysteresis loops are observed in (a), which become larger as the electric field is increased, whereas S-shaped curves are observed in (b). (c) and (d) Relationships between scaled shear stress and scaled shear rate based on the data shown in (a) and (b), respectively. The scaled shear stress and scaled shear rate are defined as $\sigma / \varepsilon_{0}|\Delta \varepsilon| E^{2}$ and $\gamma_{1} \dot{\gamma} / \varepsilon_{0}|\Delta \varepsilon| E^{2}$, respectively. The scaling relation holds for both sets of data. The solid black curve in (d) represents the theoretical result calculated by assuming the orientational distribution function.

larger than that of typically used liquid crystal cells. It was previously observed that $E_{\mathrm{c}}$ decreases with increasing gap size [28]. We performed all of our experiments at electric fields higher than $E_{\mathrm{c}}$; the minimum applied electric field in our experiments was $0.47 \mathrm{~V} / \mu \mathrm{m}$, at which we could observe spontaneous shear flow.

\section{Hysteresis loop, S-shaped curve, and scaling relation}

Figures 4(a) and 4(b) present the relationships between the shear stress $\sigma$ and the shear rate $\dot{\gamma}$ measured under controlled shear stress and controlled shear rate, respectively, at various electric field strengths, where the shear stress or shear rate was first increased from the minimum value to the maximum value and then decreased to the minimum value again. The electric field was changed at a step of $\Delta\left(E^{2}\right)=0.45(\mathrm{~V} / \mu \mathrm{m})^{2}$ and the measurement time at each of the different electric fields was $1000 \mathrm{~s}$. Under controlled shear stress, hysteresis loops were clearly observed, the size of which became larger with increasing electric field. In contrast, under controlled shear rate, S-shaped curves were obtained without hysteresis. The appearance of the curves varied because the shear stress is a single-valued function of the shear rate, whereas the latter is a multivalued function of the former. Controlling the shear rate enables us to measure the unstable region $(d \sigma / d \dot{\gamma}<0)$ and clearly recognize the occurrence of negative viscosity because the slope at the origin indicates the inverse of the viscosity $\eta_{0}$. 
Such S-shaped curves have not been observed in ferroic solids to date, because it is difficult to control the order parameters such as magnetization. Quite recently, the different behavior under controlled shear stress and rate is theoretically shown in bacterial suspensions [14].

Since negative shear viscosity is very unusual, one may ascribe it to an experimental artifact. To dispel this suspicion, we performed measurements using a different type of rheometer (ARES-G2, TA Instruments), which is a strain-controlled rheometer and can therefore be used to directly measure the shear stress regardless of whether it is positive or negative; the MCR-302 used in the above experiments is fundamentally a stress-controlled rheometer. We obtained essentially the same result as described above, thereby convincingly demonstrating the occurrence of negative viscosity (see Appendix, Sec. 2).

In Figs. 4(a) and 4(b) all of the curves in each plot have a similar appearance. From dimensional analysis we obtain the scaling relation

$$
\frac{\gamma_{1} \dot{\gamma}}{\varepsilon_{0}|\Delta \varepsilon| E^{2}}=f\left(\frac{\sigma}{\varepsilon_{0}|\Delta \varepsilon| E^{2}}\right),
$$

where $\gamma_{1}$ is the rotational viscosity $[17,18]$ and $f(x)$ is a scaling function. The scaled data from Figs. 4(a) and 4(b) are plotted in Figs. 4(c) and 4(d), respectively. The scaling relation holds for the data obtained under both controlled shear stress and controlled shear rate. It is noted for Fig. 4(b) that the slope at the origin, that is, the inverse of viscosity $\eta_{0}$, is independent of the applied electric field. This is a natural consequence of the scaling relation; since $f(x)$ in Eq. (1) is an odd function, $f(x)=f^{\prime}(0) x$ for small $x$ and thus $\gamma_{1} \dot{\gamma}=$ $f^{\prime}(0) \sigma$ independent of the applied electric field in the linear region.

\section{Temperature and frequency dependences of the S-shaped curve}

To confirm that the negative viscosity was due to the nematic phase, we examined the temperature dependence of the S-shaped curve, which would be expected to disappear in the isotropic phase. For our MBBA, the transition temperature from the nematic phase to the isotropic phase is approximately $45^{\circ} \mathrm{C}$. The experimental procedure was the same as that used in Fig. 4(b), except that the amplitude and frequency were fixed at $E=1.5 \mathrm{~V} / \mu \mathrm{m}$ and $f=50 \mathrm{~Hz}$, respectively, and the temperature was varied. As shown in Fig. 5, the viscosity $\eta_{0}$ gradually increased with increasing temperature. At $43^{\circ} \mathrm{C}$, the viscosity was positive but the shear-stress-shearrate line remained slightly curved because the sample was still in the nematic phase. At $47^{\circ} \mathrm{C}$, which corresponds to the isotropic phase, the line was straight owing to the absence of negative viscosity. This result clearly indicates that the negative viscosity originated from the nematic phase. In this case, compared with the electric field dependence [Fig. 4(b)], the behavior is different: No scaling relation holds and the viscosity $\eta_{0}$ depends on the temperature. Furthermore, the temperature dependence is reminiscent of the ferromagnetic or ferroelectric phase transition, where the hysteresis loop (S-shaped curve in our case) disappears at high temperatures in the paramagnetic or paraelectric phase. Therefore, we refer to the states with and without the S-shaped curve (hysteresis

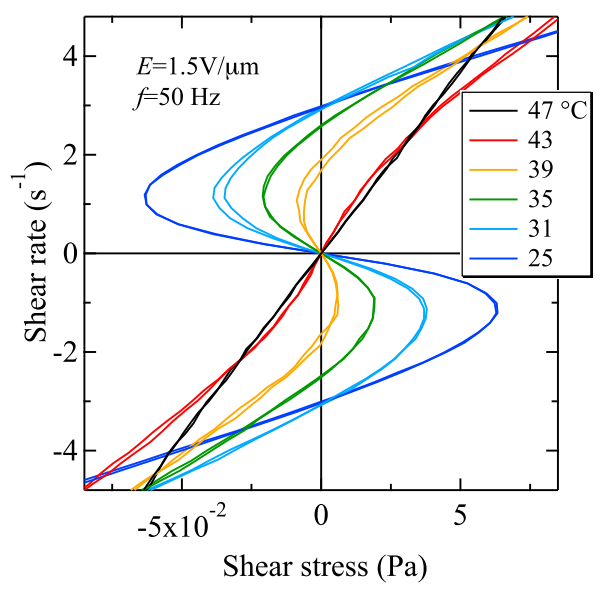

FIG. 5. Temperature dependence of the S-shaped curve. Measurements are performed under controlled shear strain at $E=$ $1.5 \mathrm{~V} / \mu \mathrm{m}$ and $f=50 \mathrm{~Hz}$. The nematic to isotropic phase transition temperature of MBBA is approximately $45^{\circ} \mathrm{C}$. At $47^{\circ} \mathrm{C}$, the nonlinear property related to the negative viscosity completely disappears.

loop) as ferroviscous and paraviscous phases, respectively. In addition, we refer to the property of spontaneous shear flow and the reversal of its direction by an external impulse, which leads to the S-shaped curve (hysteresis loop) in the ferroviscous phase, as ferroviscosity.

In general, EC is dependent on the frequency, so we can expect the S-shaped curve to be frequency dependent. Figure 6(a) shows the frequency dependence of the S-shaped curve at a constant electric field of $1.5 \mathrm{~V} / \mu \mathrm{m}$. A ferroviscous phase transition similar to that seen in Fig. 5 can be observed. In this case, the frequency plays a temperaturelike role. Both temperature and frequency are able to cause the paraviscous to ferroviscous phase transition. However, we consider that the frequency is the proper temperaturelike quantity in the ferroviscous phase transition, because the temperature is the quantity responsible for the nematic to isotropic phase transition and the frequency is related only to the ferroviscous transition. As the temperature influences various quantities such as the dielectric anisotropy and the viscosity coefficients, the temperature dependence may be more complex than the frequency dependence. We observed simple behaviors for the frequency dependence as described in the following. Figure 6(b) shows the frequency dependence of the spontaneous shear rate measured at $\sigma=0$ in Fig. 6(a), clearly indicating that the shear rate corresponds to the order parameter in conventional ferroic materials. Figure 6(c) shows the frequency dependence of the differential viscosity $d \sigma / d \dot{\gamma}$ at $\sigma=0$, where there are two other values $\eta_{+}$and $\eta_{-}$in addition to $\eta_{0}$ as indicated in Fig. 6(a); the average value $\left(\eta_{+}+\eta_{-}\right) / 2$ is plotted rather than $\eta_{+}$and $\eta_{-}$. The ferroviscous phase transition occurred at $f_{\mathrm{c}}=433 \mathrm{~Hz}$. For $\left(\eta_{+}+\eta_{-}\right) / 2\left(f<f_{\mathrm{c}}\right)$ and $\eta_{0}\left(f>f_{\mathrm{c}}\right)$, the typical Curie-Weiss law holds, so they correspond to the inverse magnetic susceptibility, and the absolute value of the slope for the former is approximately 1.8 times as large as that for the latter, which is close to the value of 2 calculated from the mean-field theory or Landau theory [31] for ferroic materials. In addition, negative $\eta_{0}\left(f<f_{\mathrm{c}}\right)$ was successfully observed, unlike in conventional ferroic materials. 

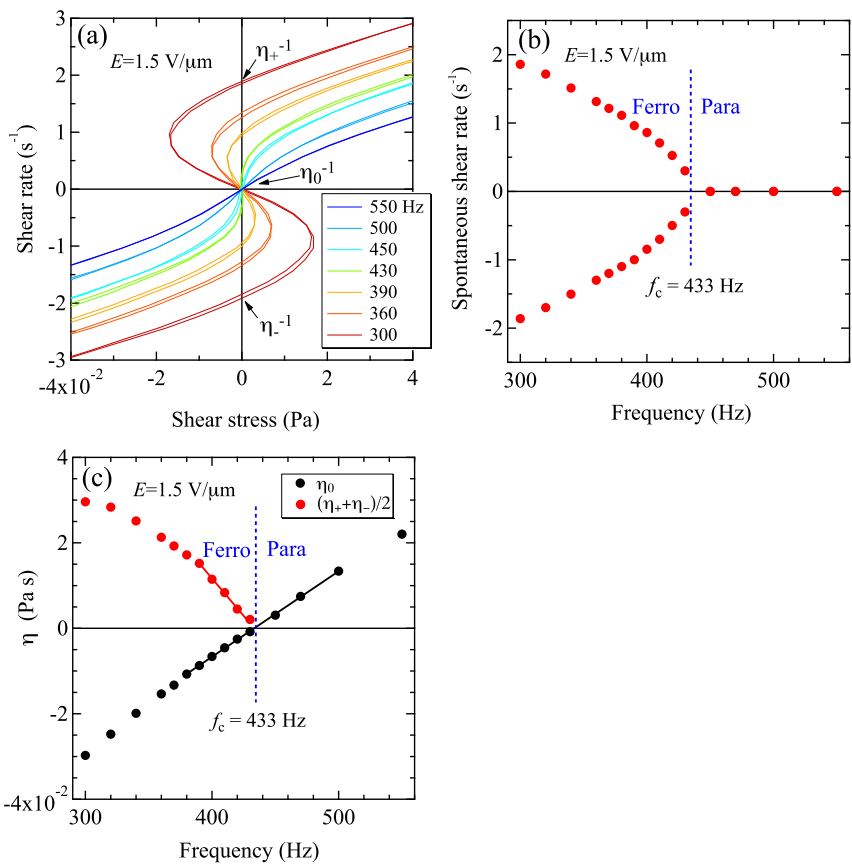

FIG. 6. Frequency-induced transition from the paraviscous phase to the ferroviscous phase. (a) Frequency dependence of the S-shaped curve at $E=1.5 \mathrm{~V} / \mu \mathrm{m}$. At high frequencies, the slope at $\dot{\gamma}=0$ is positive, which corresponds to the paraviscous phase. Therefore, the transition from the ferroviscous phase to the paraviscous phase can be observed. (b) Frequency dependence of the spontaneous shear rate at zero shear stress obtained from (a). A steep increase in the spontaneous shear rate is observed below the transition point, which is similar to the order parameter of conventional ferroic materials. (c) Frequency dependence of differential viscosity $d \sigma / d \dot{\gamma}$ at $\sigma=0$, showing $\eta_{0}$ and the average $\left(\eta_{+}+\eta_{-}\right) / 2$. At the transition point of $f_{\mathrm{c}}=433 \mathrm{~Hz}, \eta_{0}$ becomes zero.

\section{E. Self-oscillation caused by negative viscosity}

Negative electrical resistance can be used to produce electrical oscillators, which are based on negative-resistance devices such as tunnel diodes [32]. In exactly the same manner, we can construct a mechanical oscillator simply by attaching a coil spring to the shaft of the upper plate (Fig. 7). With the spring, the equation of motion for the rotational angle $\phi$ becomes

$$
I \ddot{\phi}=-K \phi-\Gamma \dot{\phi}-M(\dot{\phi}),
$$

where $I, K$, and $\Gamma$ are the moment of inertia of the upper disk, the spring constant of the coil spring, and the damping constant of the coil spring excluding the viscosity of the liquid crystal, respectively. In addition, $M(\dot{\phi})$ is the torque exerted on the liquid crystal for an angular velocity $\dot{\phi}$, which is obtained from the S-shaped curve shown in Fig. 4(b), $M=$ $\left(\pi r^{3} / 2\right) \sigma$ and $\dot{\phi}=(h / r) \dot{\gamma}$, where $r$ and $h$ are the radius of the upper disk and the gap, respectively. When $\Gamma \dot{\phi}+M(\dot{\phi})=$ $a_{1} \dot{\phi}+a_{3} \dot{\phi}^{3}$, Eq. (2) is the Rayleigh equation [33], which is transformed, by differentiating it with respect to time, to the van der Pol equation [34]

$$
I \ddot{\Phi}=-K \Phi-\left(a_{1}+3 a_{3} \Phi^{2}\right) \dot{\Phi},
$$

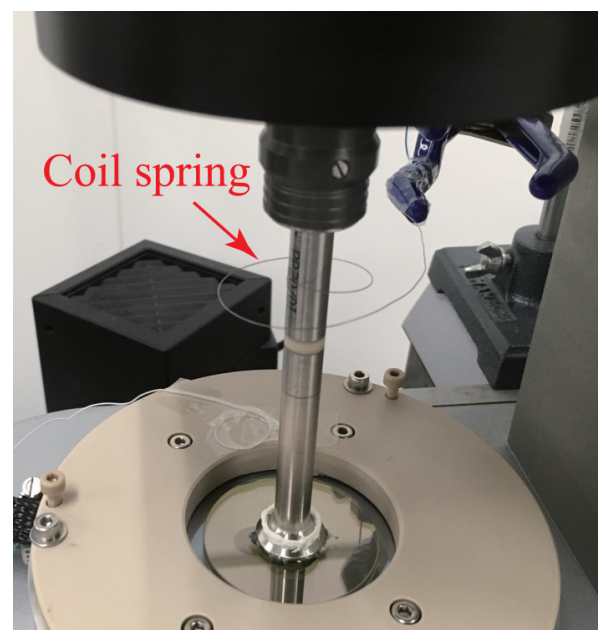

FIG. 7. Mechanical self-oscillator. A coil spring is simply attached to the shaft of the upper plate of the rheometer.

where $\Phi=\dot{\phi}$. For $a_{1}<0$, the static solution is unstable and a limit cycle appears. In our system, we can demonstrate a selfoscillation, although $M(\dot{\phi})$ contains higher-order terms with respect to $\dot{\phi}$ in this case.

Figure 8(a) shows a typical self-oscillation observed in the angular velocity $\dot{\phi}$ after applying an electric field of $E=1.5 \mathrm{~V} / \mu \mathrm{m}$ and $f=50 \mathrm{~Hz}$. Here we confirm that the self-oscillation originates from the $\mathrm{S}$-shaped curve according to Eq. (2). Figure 9 shows the S-shaped curve in the $M$ and $\dot{\phi}$ plane, which was measured by removing the coil spring after observing the self-oscillation in Fig. 8(a). To numerically solve Eq. (2), we assume an odd function for $M(\dot{\phi})$ as

$$
M(x)=-a x+b x|x|^{1.5} /\left(|x|^{1.5}+c^{1.5}\right),
$$

where $a, b$, and $c$ are fitting parameters, which were obtained from Fig. 9 by least-squares fitting. In our data for $M(\dot{\phi})$, polynomial functions are not suitable for fitting. The black line in Fig. 9 shows the fitting result. Here the fitting range was chosen such that it covers the moving range of $\dot{\phi}$ in Fig. 8(a).
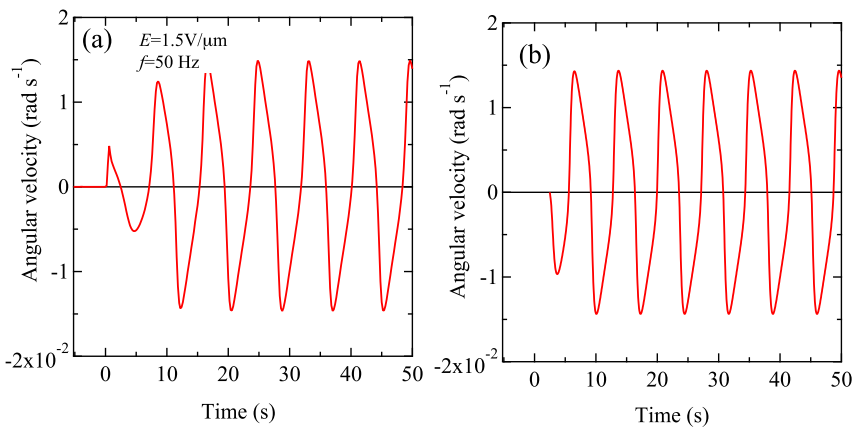

FIG. 8. Mechanical self-oscillation of angular velocity. (a) Experimental result observed upon applying an ac electric field with an amplitude of $1.5 \mathrm{~V} / \mu \mathrm{m}$ and a frequency of $50 \mathrm{~Hz}$ at $t=0$. A self-oscillation is observed in both the angular velocity $\dot{\phi}$ and the rotation angle $\phi$. (b) Numerical result calculated using Eq. (2). The initial condition of $\phi=2.98 \times 10^{-3} \mathrm{rad}$ and $\dot{\phi}=0 \mathrm{rad} \mathrm{s}^{-1}$ was imposed at $t=2.47 \mathrm{~s}$; these values were obtained from the data of $\phi(t)$ (not shown) and (a). 


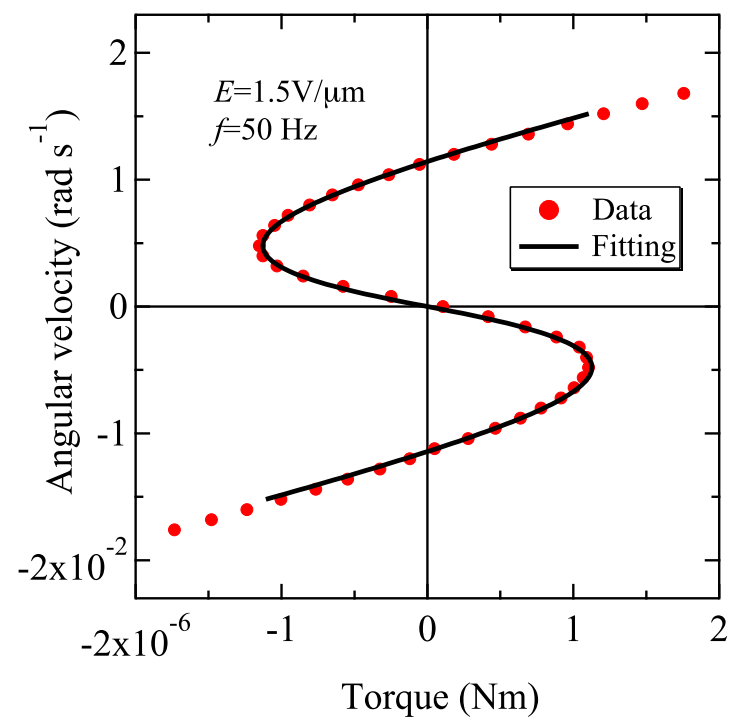

FIG. 9. Plot of the S-shaped curve. This curve was measured without the coil spring shortly after observing the self-oscillation. The applied electric field was the same as that used to obtain Fig. 8. The average of the data obtained while increasing and decreasing the shear rate is shown. The solid black line indicates the fitting result from Eq. (4) with $a=4.58 \times 10^{-4} \mathrm{Nms} \mathrm{rad}^{-1}, b=$ $7.56 \times 10^{-4} \mathrm{Nms} \mathrm{rad}^{-1}$, and $c=7.97 \times 10^{-3} \mathrm{~s}^{-1}$.

The moment of inertia $I$ was measured using the rheometer. The parameters $K$ and $\Gamma$ were obtained from a damped oscillation without the liquid crystal sample, as shown in Fig. 10, where we fitted the function $\phi(t)=A \exp (-t / \tau) \cos (\omega t+\delta)$ to the data to obtain $K=I\left(\omega^{2}+\tau^{-2}\right)$ and $\Gamma=2 I \tau^{-1}$. Using these obtained parameters, we determined $\dot{\phi}(t)$ by numerically solving Eq. (2). As shown in Fig. 8(b), we were able to successfully reproduce the experimental data, which convincingly demonstrates that the self-oscillation originated from

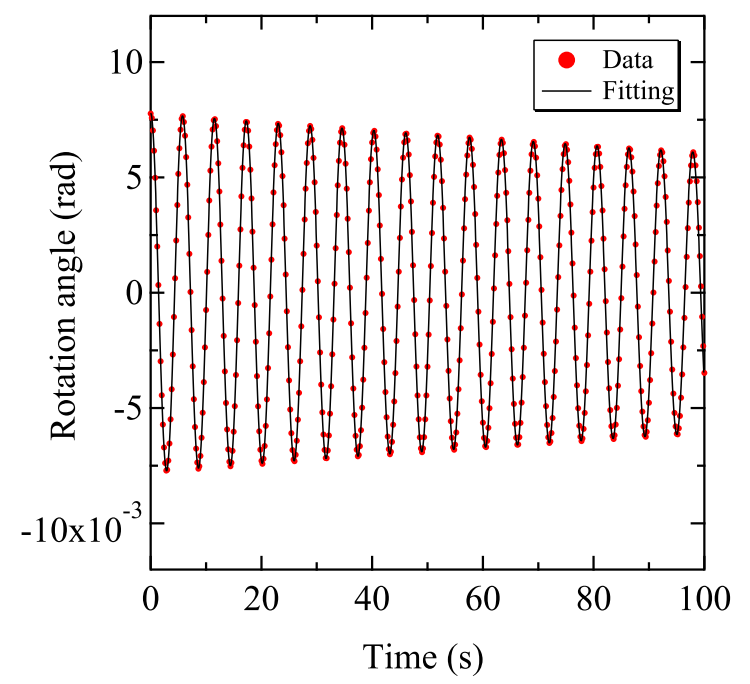

FIG. 10. Damped oscillation. Time dependence of the rotational angle $\phi$ after applying an initial rotational angle of $7.8 \times$ $10^{-3} \mathrm{rad}$ with the coil spring and no liquid crystal. From the leastsquares fit, we obtain $K=1.15 \times 10^{-4} \mathrm{Nm} \mathrm{rad}^{-1}$ and $\Gamma=4.6 \times$ $10^{-7} \mathrm{Nms} \mathrm{rad}^{-1}$ by using $I=9.70 \times 10^{-5} \mathrm{Nms}^{2} \mathrm{rad}^{-1}$.

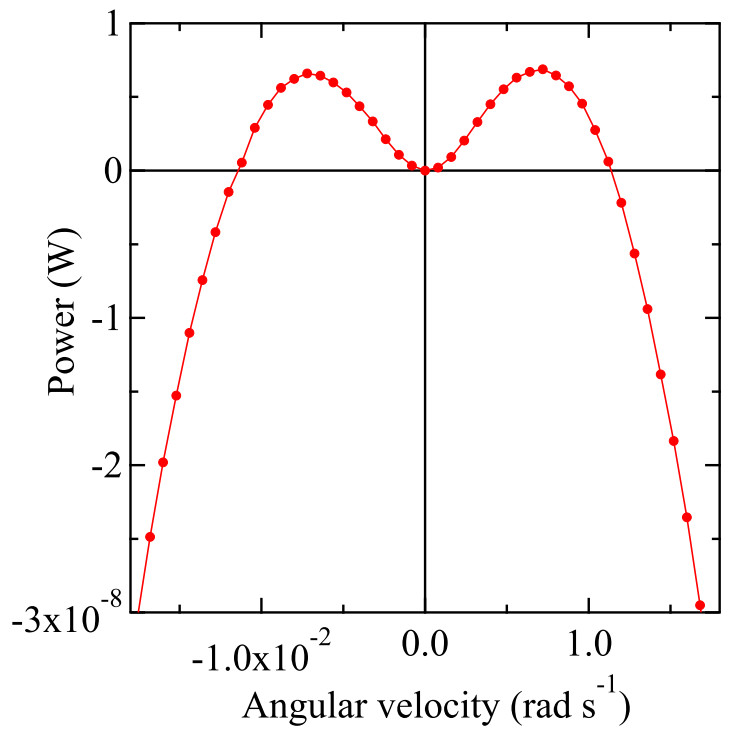

FIG. 11. Power density $-M \dot{\phi}$ as a function of $\dot{\phi}$ obtained from the S-shaped curve shown in Fig. 9. The maximum power density is approximately $6.7 \times 10^{-9} \mathrm{~W}$ at $\dot{\phi} \simeq \pm 7.2 \times 10^{-3} \mathrm{rad} \mathrm{s}^{-1}$ and $M \simeq \mp 9.3 \times 10^{-7} \mathrm{Nm}$.

the negative and nonlinear viscosity depicted in Fig. 9. The amplitudes of oscillation for the experimental and numerical results were 0.015 and $0.014 \mathrm{rad} \mathrm{s}^{-1}$, respectively, and the periods were 8.2 and $7.2 \mathrm{~s}$, respectively. The discrepancy, especially with respect to the period, may be attributable to a dynamical effect; the $M-\dot{\phi}$ curve in Fig. 9, which was statically measured, could be changed by a faster measurement made, for example, within the period.

Finally, we briefly consider the efficiency when we regard the system with no coil spring, which spontaneously rotates, as a motor. From the S-shaped curve in Fig. 9, the work done on the upper disk by the liquid crystal per unit time, that is, the power $-M \dot{\phi}$, was calculated as a function of the angular velocity (Fig. 11). The maximum value of the power was about $6.7 \times 10^{-6} \mathrm{~mW}$ at $\dot{\phi} \simeq \pm 7.2 \times 10^{-3} \mathrm{rad} \mathrm{s}^{-1}$ and $M \simeq$ $\mp 9.3 \times 10^{-7} \mathrm{Nm}$, which is much smaller than the supplied electric power of $20 \mathrm{~mW}$.

\section{DISCUSSION}

\section{A. Origin of negative viscosity and separation of viscous and electric-field-induced stresses}

We next consider the origin of the negative viscosity. Upon the application of an electric field, macroscopic shear flow is generated by the rotation of the director caused by the electrically induced torque, which may generate rotational flows and reduce the apparent viscosity. This effect appears to be similar to that in MFs. However, there is an essential difference between the two systems. In MFs, each particle possesses a permanent magnetic moment, which rotates according to an applied ac magnetic field and induces a vortex flow around the particle. The rotational directions are usually random such that no net macroscopic flow is present. However, any shear flow can break down the degeneracy of the rotational direction, and the resultant synchronous rotation reduces the 
apparent viscosity. In MFs, the magnetic moment is a vector and tends to be parallel to the applied magnetic field, whereas in NLCs the director is a second-rank-tensor-like quantity and tends to be perpendicular to the electric field for NLCs with negative dielectric anisotropy. In the former case, reversal of the magnetic moment or $\pi$ rotation of the particle is possible by applying a magnetic field in the opposite direction, whereas in the latter case this is impossible. This means that in NLCs, it is difficult to make the director rotate synchronously with the ac electric field and therefore it is necessary to find another mechanism to explain the negative viscosity. In fact, we observed spontaneous shear flow even under dc electric fields (the spontaneous shear rate was $2.7 \mathrm{~s}^{-1}$ at a $\mathrm{dc}$ electric field of $1.06 \mathrm{~V} / \mu \mathrm{m})$, which definitively rules out the above mechanism based on synchronous rotation with an ac electric field. In the present NLC, however, turbulence occurs, which induces random director reorientation through the coupling between the flow and director. In this state, an electric torque is exerted on the director when the director is not perpendicular to the applied electric field. Consequently, the torque gives the director electrically induced angular velocity, resulting in the generation of a local flow. When the local flow synchronizes over the fluid, the apparent viscosity is reduced as in the case of MFs. This scenario is formulated based on the Ericksen-Leslie (EL) theory $[17,18]$.

In EL theory, the stress tensor $\sigma_{\alpha \beta}$ is expressed in terms of the director field $\mathbf{n}(\mathbf{x}, t)$ and velocity field $\mathbf{v}(\mathbf{x}, t)$,

$$
\sigma_{\alpha \beta}=\sigma_{\alpha \beta}^{(\text {Ericksen })}+\sigma_{\alpha \beta}^{(\text {visc })}
$$

with

$$
\sigma_{\alpha \beta}^{(\text {Ericksen })}=-\frac{\partial f_{d}}{\partial\left(\partial n_{\gamma} / \partial x_{\alpha}\right)} \frac{\partial n_{\gamma}}{\partial x_{\beta}}-p \delta_{\alpha \beta}
$$

and

$$
\begin{aligned}
\sigma_{\alpha \beta}^{(\text {visc })}= & \alpha_{4} A_{\alpha \beta}+\alpha_{1} n_{\alpha} n_{\beta} n_{\mu} n_{\rho} A_{\mu \rho} \\
& +\alpha_{5} n_{\alpha} n_{\mu} A_{\mu \beta}+\alpha_{6} n_{\beta} n_{\mu} A_{\mu \alpha} \\
& +\alpha_{2} n_{\alpha} N_{\beta}+\alpha_{3} n_{\beta} N_{\alpha},
\end{aligned}
$$

where $f_{d}$ is the Frank elastic energy density, $p$ is the pressure, $A_{\alpha \beta}=\left(\partial v_{\alpha} / \partial x_{\beta}+\partial v_{\beta} / \partial x_{\alpha}\right) / 2$ is the symmetric part of the velocity gradient tensor, $N_{\alpha}=d n_{\alpha} / d t-W_{\alpha \beta} n_{\beta}$ is the rate of change of the director with respect to the background fluid with the antisymmetric part $W_{\alpha \beta}=\left(\partial v_{\alpha} / \partial x_{\beta}-\partial v_{\beta} / \partial x_{\alpha}\right) / 2$, and $\alpha_{i}$ are the Leslie viscosity coefficients. In addition, $\sigma_{\alpha \beta}^{(\text {Ericksen) }}$ is the Ericksen stress, the first term of which originates from the elasticity, and $\sigma_{\alpha \beta}^{\text {(visc) }}$ is the viscous stress due to dissipation. In our case, we can neglect the Ericksen stress. The elastic energy density is given by

$$
\begin{aligned}
f_{d}= & \frac{1}{2} K_{1}(\nabla \cdot \mathbf{n})^{2}+\frac{1}{2} K_{2}[\mathbf{n} \cdot(\nabla \times \mathbf{n})]^{2} \\
& +\frac{1}{2} K_{3}[\mathbf{n} \times(\nabla \times \mathbf{n})]^{2},
\end{aligned}
$$

where $K_{i}$ are the Frank elastic constants. Using this expression, we can estimate the Ericksen stress as $K h^{-2}$, where $K$ and $h$ are a typical elastic constant of MBBA and the gap height, respectively. Substituting $K \simeq 5 \times 10^{-12} \mathrm{~N}$ [17] and $h=100 \mu \mathrm{m}$, we obtain $K h^{-2} \simeq 5 \times 10^{-4} \mathrm{~Pa}$, which is much smaller than the values measured from Fig. 4. Hereafter, we consider only the viscous stress. In Eq. (7), the two terms containing $N_{\alpha}$ may be the origin of the negative viscosity because they are related to the stress generated by the angular velocity of the director.

The $N_{\alpha}$ is calculated from the torque balance equation

$$
\mathbf{n} \times \mathbf{h}=\gamma_{1} \mathbf{n} \times \mathbf{N}+\gamma_{2} \mathbf{n} \times A \mathbf{n},
$$

where $\mathbf{h}=-\partial f_{\mathrm{el}} / \partial \mathbf{n}=\varepsilon_{0} \Delta \varepsilon(\mathbf{n} \cdot \mathbf{E}) \mathbf{E}$ is the force density originating from the electric field, $\gamma_{1}=\alpha_{3}-\alpha_{2}$, and $\gamma_{2}=$ $\alpha_{3}+\alpha_{2}[17,18]$. By using the fact that $\mathbf{N}$ is perpendicular to $\mathbf{n}$, which is derived from its definition, together with Eq. (9), we obtain

$$
\mathbf{N}=-\frac{\gamma_{2}}{\gamma_{1}}\{\mathbf{A n}-(\mathbf{n} \cdot \mathbf{A n}) \mathbf{n}\}+\frac{1}{\gamma_{1}} \varepsilon_{0} \Delta \varepsilon(\mathbf{n} \cdot \mathbf{E})\{\mathbf{E}-(\mathbf{n} \cdot \mathbf{E}) \mathbf{n}\} .
$$

Substitution of Eq. (10) into Eq. (7) yields

$$
\begin{aligned}
\sigma_{\alpha \beta}^{(\text {visc })}= & \alpha_{4} A_{\alpha \beta}+\left\{\alpha_{1}+\frac{\gamma_{2}}{\gamma_{1}}\left(\alpha_{2}+\alpha_{3}\right)\right\} n_{\alpha} n_{\beta} n_{\mu} n_{\rho} A_{\mu \rho} \\
& +\left(\alpha_{5}-\frac{\gamma_{2}}{\gamma_{1}} \alpha_{2}\right) n_{\alpha} n_{\mu} A_{\mu \beta}+\left(\alpha_{6}-\frac{\gamma_{2}}{\gamma_{1}} \alpha_{3}\right) n_{\beta} n_{\mu} A_{\mu \alpha} \\
& +\frac{1}{\gamma_{1}} \varepsilon_{0} \Delta \varepsilon\left\{\alpha_{2} n_{\alpha} n_{\mu} E_{\beta} E_{\mu}+\alpha_{3} n_{\beta} n_{\mu} E_{\alpha} E_{\mu}\right. \\
& \left.-\left(\alpha_{2}+\alpha_{3}\right) n_{\alpha} n_{\beta} n_{\mu} n_{\rho} E_{\mu} E_{\rho}\right\} .
\end{aligned}
$$

Since our fluid is in a turbulent state, we take the time and spatial average. By using the decoupling approximation $\left\langle n_{\alpha} n_{\beta} n_{\mu} n_{\rho} A_{\mu \rho}\right\rangle=\left\langle n_{\alpha} n_{\beta} n_{\mu} n_{\rho}\right\rangle\left\langle A_{\mu \rho}\right\rangle$ for the turbulent state, for a macroscopic shear flow $\mathbf{v}=(\dot{\gamma} z, 0,0)$ and an electric field $\mathbf{E}=(0,0, E)$, the measured shear stress $\sigma=\left\langle\sigma_{z x}^{(\text {visc })}\right\rangle$ can be expressed as

$$
\sigma=\sigma_{\mathrm{v}}+\sigma_{\mathrm{e}}
$$

with

$$
\begin{aligned}
\sigma_{\mathrm{v}}= & \frac{\dot{\gamma}}{2}\left[\alpha_{4}+2\left\{\alpha_{1}+\frac{\gamma_{2}}{\gamma_{1}}\left(\alpha_{2}+\alpha_{3}\right)\right\}\left\langle n_{x}^{2} n_{z}^{2}\right\rangle\right. \\
& \left.+\left(\alpha_{5}-\frac{\gamma_{2}}{\gamma_{1}} \alpha_{2}\right)\left\langle n_{z}^{2}\right\rangle+\left(\alpha_{6}-\frac{\gamma_{2}}{\gamma_{1}} \alpha_{3}\right)\left\langle n_{x}^{2}\right\rangle\right]
\end{aligned}
$$

and

$$
\sigma_{\mathrm{e}}=\varepsilon_{0} \Delta \varepsilon E^{2} \frac{1}{\gamma_{1}}\left[\alpha_{3}\left\langle n_{x} n_{z}\right\rangle-\left(\alpha_{2}+\alpha_{3}\right)\left\langle n_{x} n_{z}^{3}\right\rangle\right] .
$$

Here we take the $x, y$, and $z$ axes along the flow, vorticity, and velocity gradient (electric field) directions, respectively [Fig. 1(b)], and $E$ in Eq. (14) should be regarded as the root-mean-square value as the time average is taken. Although both $\sigma_{\mathrm{v}}$ and $\sigma_{\mathrm{e}}$ are derived from the viscous stress, we refer to the former as the intrinsic viscous stress or simply the viscous stress, because it persists even in the absence of an electric field. We refer to $\sigma_{\mathrm{e}}$ as the electric-field-induced shear stress, as it is directly related to the electric field. It should be noted that these two stresses are expressed only in terms of the averages of products of $n_{\alpha}$ and contain no angular velocity. This is one advantage of our formulation. Without shear flow in the $x-z$ plane, the orientational distribution of the director is rotationally symmetric with respect to the $z$ axis, resulting in $\left\langle n_{x} n_{z}\right\rangle=\left\langle n_{x} n_{z}^{3}\right\rangle=0$ in Eq. (14), and therefore $\sigma_{\mathrm{e}}=0$. 

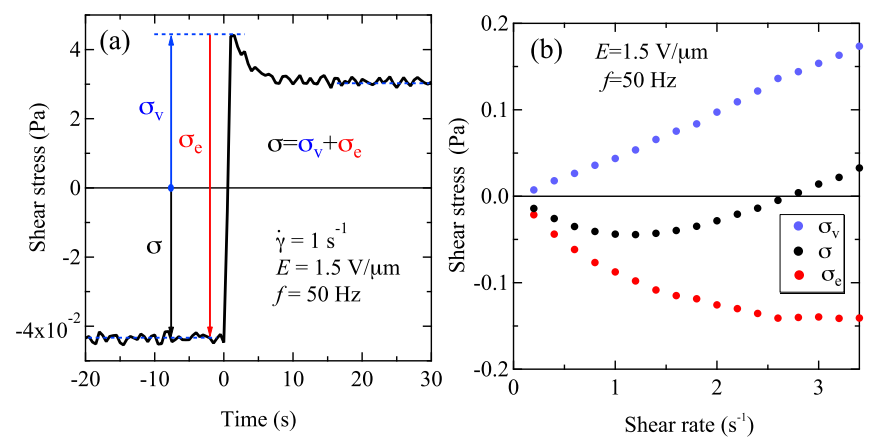

FIG. 12. Separation of $\sigma_{\mathrm{v}}$ and $\sigma_{\mathrm{e}}$. (a) Transient response of shear stress at a shear rate of $1 \mathrm{~s}^{-1}$ upon removing an electric field of $1.5 \mathrm{~V} / \mu \mathrm{m}$ at $0 \mathrm{~s}$. The stress suddenly increases immediately after removal of the electric field, corresponding to $-\sigma_{\mathrm{e}}>0$, and then slowly relaxes. The shear stress prior to removal of the electric field is $\sigma_{\mathrm{v}}+\sigma_{\mathrm{e}}$. From these values, we can separately obtain $\sigma_{\mathrm{v}}$ and $\sigma_{\mathrm{e}}$. (b) Dependences of $\sigma_{\mathrm{v}}$ and $\sigma_{\mathrm{e}}$ on $\dot{\gamma}$ obtained by varying the shear rate in (a). Here $\sigma_{\mathrm{v}}$ is positive and nearly proportional to the shear rate, whereas $\sigma_{\mathrm{e}}$ is negative and becomes saturated at high shear rates. Therefore, $\sigma=\sigma_{\mathrm{v}}+\sigma_{\mathrm{e}}$ first decreases to a minimum and then increases to become positive. This behavior corresponds to part of Fig. 4(b) $(\dot{\gamma}>0)$.

Any shear flow can break down the symmetry to make the averages nonzero. If $\sigma_{\mathrm{e}}<0$, the apparent viscosity decreases, and furthermore, if $-\sigma_{\mathrm{e}}>\sigma_{\mathrm{v}}$, it becomes negative.

We can experimentally separate $\sigma_{\mathrm{v}}$ and $\sigma_{\mathrm{e}}$ using the fact that $\sigma_{\mathrm{e}}$ disappears when the electric field is removed, as indicated by Eq. (14). Figure 12(a) shows the time dependence of the shear stress at a shear rate of $1 \mathrm{~s}^{-1}$ upon removing an electric field of $1.5 \mathrm{~V} / \mu \mathrm{m}$ at $0 \mathrm{~s}$. The stress suddenly increased immediately after the removal of the electric field, corresponding to $-\sigma_{\mathrm{e}}>0$. The gradual decrease after the sudden increase can be ascribed to the change in the orientational distribution. We repeated this measurement for various shear rates to obtain the shear-rate dependences of $\sigma_{\mathrm{v}}$ and $\sigma_{\mathrm{e}}$ [Fig. 12(b)]. It can be seen that $\sigma_{\mathrm{v}}$ was positive and nearly proportional to $\dot{\gamma}$, whereas $\sigma_{\mathrm{e}}$ was negative and became saturated at high shear rates. These behaviors are consistent with Eqs. (13) and (14); the average values of the product of $n_{\alpha}$ in Eq. (13) are nonzero even in the absence of an electric field and therefore they may be almost independent of the electric field, whereas those in Eq. (14) are zero in the absence of an electric field and so an increase would be expected when an electric field is applied. Thus, the electric-field-induced shear stress is clearly confirmed to be the origin of the negative stress in the present NLC system.

\section{B. Model to reproduce the S-shaped curve}

We can now reproduce the $S$-shaped curves by calculating the averages of the products of $n_{\alpha}$ in Eqs. (13) and (14). To do so, we assume the orientational distribution function as

$$
P(\mathbf{n}) \propto \exp \left(-a \frac{1}{2} n_{z}^{2}+\frac{b \tilde{\dot{\gamma}} \tilde{\dot{\gamma}}_{0}}{\sqrt{\tilde{\dot{\gamma}}^{2}+\tilde{\dot{\gamma}}_{0}^{2}}} n_{x} n_{z}\right),
$$

where $\widetilde{\dot{\gamma}}$ is the scaled shear rate

$$
\widetilde{\gamma}=\frac{\gamma_{1} \dot{\gamma}}{\varepsilon_{0}|\Delta \varepsilon| E^{2}}
$$

and $a$ and $b$ are constants. Here we regard the turbulence as a heat bath in analogy to equilibrium systems. The first term in the exponential function originates from the interaction energy with the electric field $f_{\mathrm{el}}=-\frac{1}{2} \varepsilon_{0} \varepsilon_{\perp} E^{2}-$ $\frac{1}{2} \varepsilon_{0} \Delta \varepsilon(\mathbf{n} \cdot \mathbf{E})^{2}$, which is rotationally symmetrical with respect to the $z$ axis. The second term represents the symmetry breaking due to the macroscopic shear flow. Here we introduce a characteristic scaled shear rate $\widetilde{\dot{\gamma}}_{0}$ over which the second term becomes constant to obtain a better fitting result. Furthermore, the scaling relation expressed by Eq. (1) is considered, which indicates that the orientational distribution function should contain only the scaled shear rate $\widetilde{\dot{\gamma}}$, but not $\dot{\gamma}$ and $E$ individually. We obtain $\sigma$ using Eqs. (13)-(15), where we replace the external electric field $E$ in Eq. (14) by the root mean square $E / \sqrt{2}$ (note that $E$ represents the amplitude of the ac electric field in this paper) and modify Eq. (12) to $\sigma=$ $c\left(\sigma_{\mathrm{v}}+\sigma_{\mathrm{e}}\right)$ with a constant $c$ to improve the fitting. Since the calculated $\sigma$ is not so sensitive to the parameter $a$, we set $a=$ 0 for simplicity. The parameters $b, c$, and $\tilde{\dot{\gamma}}_{0}$ are adjustable, and literature data are used for the viscosity coefficients $\left(\alpha_{1}=6 \mathrm{mPa} \mathrm{s}, \alpha_{2}=-77.4 \mathrm{mPa} \mathrm{s}, \alpha_{3}=-0.868 \mathrm{mPa} \mathrm{s}, \alpha_{4}=\right.$ $81.8 \mathrm{mPa} \mathrm{s}, \alpha_{5}=57.2 \mathrm{mPa} \mathrm{s}$, and $\alpha_{6}=-32.5 \mathrm{mPa} \mathrm{s}$ [35]) and the dielectric anisotropy $\left(\Delta \varepsilon=-0.631\right.$ [36]) at $25^{\circ} \mathrm{C}$. The former parameters were determined for $E=1.34 \mathrm{~V} / \mu \mathrm{m}$ as $b=0.76, c=1.6$, and $\widetilde{\dot{\gamma}_{0}}=0.011$ from the least-squares fit. The corresponding theoretical curve is indicated by the black line in Fig. 4(d). The orientational distribution for this case is depicted in Fig. 13, which shows the surface plot of $P(\mathbf{n})$ n. In the absence of shear flow, $\widetilde{\dot{\gamma}}=0$, the distribution is isotropic because we set $a=0$, although in general it should be rotationally symmetrical with respect to the $z$ axis
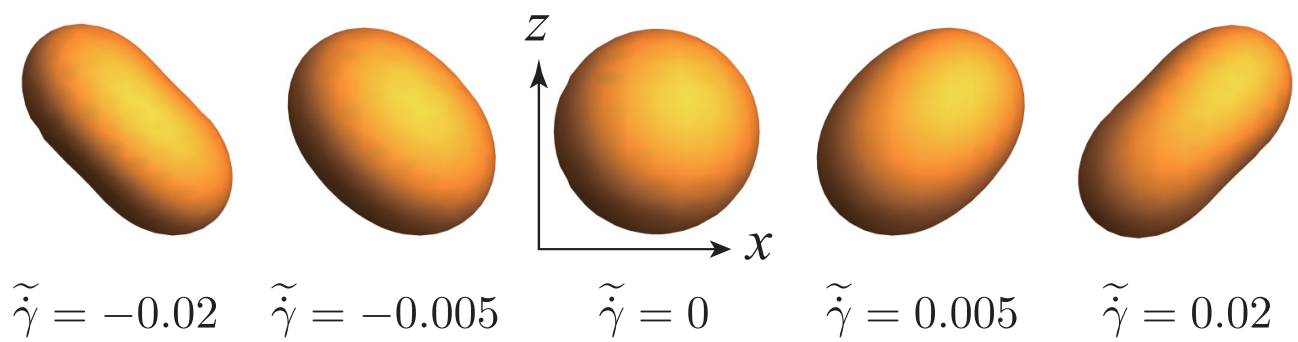

$$
\tilde{\dot{\gamma}}=0 \quad \tilde{\dot{\gamma}}=0.005
$$

$$
\tilde{\dot{\gamma}}=0.02
$$

FIG. 13. Shear rate dependence of orientational distribution. The surface plot of $P(\mathbf{n}) \mathbf{n}$ was calculated from Eq. (5) with $a=0, b=0.76$, and $\widetilde{\tilde{\gamma}}_{0}=0.011$ obtained from the fitting for $E=1.34 \mathrm{~V} / \mu \mathrm{m}$. Symmetry breaking is observed in the presence of shear flow as expected. 

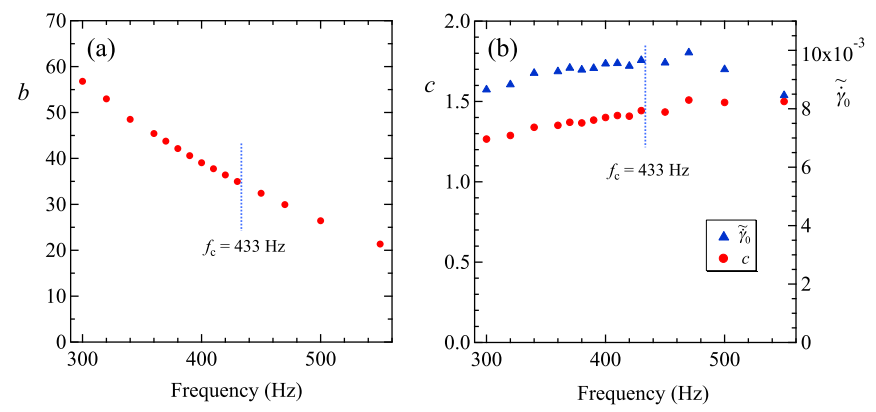

FIG. 14. Frequency dependences of (a) $b$ and (b) $c$ and $\widetilde{\gamma}_{0}$ obtained from the fitting of Fig. 6(a). The parameter $b$ linearly decreases with frequency, whereas $c$ and $\widetilde{\dot{\gamma}}_{0}$ are almost independent of frequency.

as mentioned above. In contrast, in the presence of shear flow, the symmetry is broken and the anisotropy increases with the shear rate. In addition, the tilt of the distribution depends on the flow direction.

In the model described above, the so-called eddy viscosity [37-39] due to turbulence is not considered; this may partially contribute to the negative viscosity and thus its inclusion may be necessary to fully understand the mechanism underlying the negative viscosity in the present system. The fact that $c \neq$ 1 may indicate that the eddy viscosity contributes somewhat to the negative viscosity. This may be also ascribed to the approximation used in the derivation of Eqs. (12)-(14).

Finally, we discuss the frequency dependences of $b, c$, and $\widetilde{\gamma}_{0}$ obtained from the fitting of Fig. 6(a). As shown in Fig. 14(a), the parameter $b$ linearly decreased with frequency without any anomaly observed at the transition point from the ferroviscous phase to the paraviscous phase at $f_{\mathrm{c}}=433 \mathrm{~Hz}$. It can be seen from the frequency dependences of $\eta_{0}$ [Fig. 6(c)] and $b$ [Fig. 14(a)] that there is a close relation between them. From Eqs. (12) and (15) we obtain a simple relation

$$
\eta_{0}=\left(\frac{\alpha_{4}}{2}+\frac{\alpha_{5}}{6}+\frac{\alpha_{6}}{6}+\frac{\alpha_{1}}{15}-\frac{\alpha_{2}+\alpha_{3}}{10} \frac{\gamma_{2}}{\gamma_{1}}\right)+\frac{3 \alpha_{2}-4 \alpha_{3}}{210} b .
$$

Note that this equation is independent of the electric field, as would be expected from the scaling relation. On the other hand, $c$ and $\widetilde{\dot{\gamma}}_{0}$ are almost independent of the frequency [Fig. 14(b)]. Thus, Eq. (15) is demonstrated to be a good function for expressing the orientational distribution, although it should be derived from, for example, the Fokker-Planck equation. It is expected that there is irreversible circulation of probability current [40] in the director space (the surface of a unit sphere), which is one of the most remarkable characteristics of nonequilibrium steady states.

\section{CONCLUSION}

We have demonstrated that an NLC exhibits enormous negative viscosity in the presence of turbulence when subjected to electric fields. Several phenomena originating from the negative viscosity were observed. In the shear-stress-shearrate curves, hysteresis loops were observed under controlled shear stress, whereas S-shaped curves, which clearly illustrate

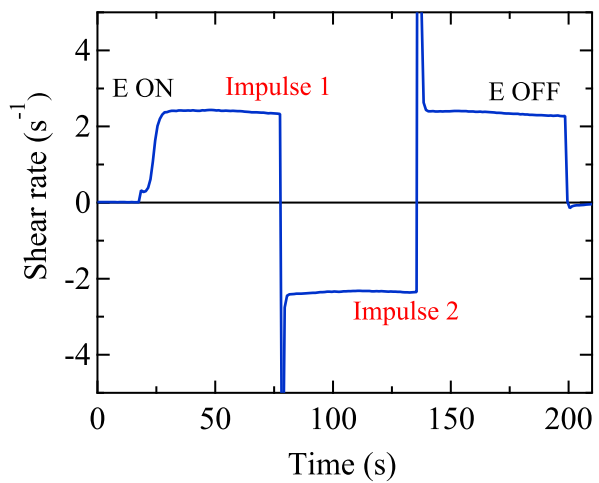

FIG. 15. Time dependence of spontaneous shear rate corresponding to video 1 in [30], upon applying impulsive torques to the upper disk.

the occurrence of negative viscosity, were observed under controlled shear rate. These hysteresis loops are quite similar to those of ferroic materials, and the similarities between the phenomena observed for our system and ferroic materials were demonstrated. By analogy with ferroic materials, we have coined the terms ferroviscosity, ferroviscous, and paraviscous. However, the ferroic materials are at equilibrium, whereas our fluid is out of equilibrium and electric energy is constantly being supplied and partially transformed to the spontaneous rotation and oscillation of the upper disk. Theoretical consideration based on the Ericksen-Leslie theory revealed that the negative viscosity originates from the electricfield-induced shear stress, which is generated by the rotation of the director in the presence of turbulence. Although a simple model has been presented that reproduces the S-shaped curve, the orientational distribution function has not yet been theoretically derived and further issues such as the mechanism of the turbulence generation remain open. Ferroviscous fluids are expected to become an important subject of research in nonequilibrium physics with potential applications to small microfluidic devices.

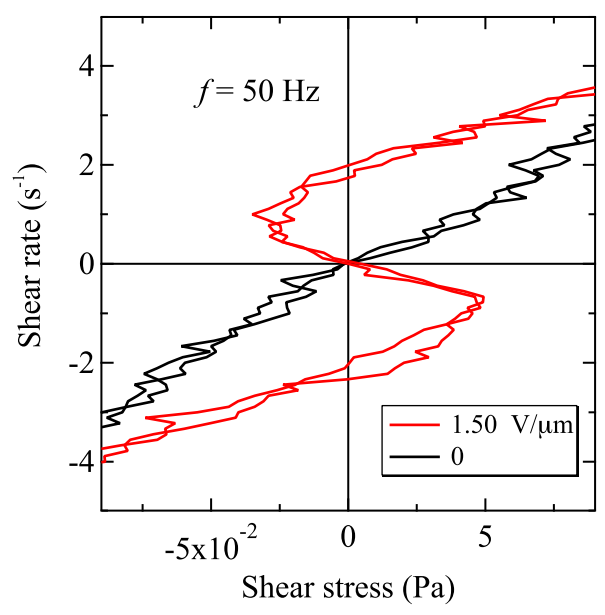

FIG. 16. Shear-rate-shear-stress curve measured using a straincontrolled rheometer. 


\section{ACKNOWLEDGMENTS}

The authors would like to thank Yoshiki Hidaka for stimulating discussions. T.N. and Y.G. thank Shigetoshi Nara for valuable comments. This work was supported by JSPS KAKENHI Grants No. JP25103006, No. JP26289032, No. JP15K13553, and No. JP18H01374.

H.O. and T.N. contributed equally to this work.

\section{APPENDIX}

\section{Spontaneous rotation of the upper disk and reversal of the rotational direction}

When an ac electric field ( $E=1.5 \mathrm{~V} / \mu \mathrm{m}$ and $f=50 \mathrm{~Hz}$ ) is applied, the upper disk starts to rotate (see video 1 in [30]). In the case of our system, the disk rotated clockwise, but the sense is not predetermined. By applying an impulsive torque in the opposite direction to the shaft of the upper disk using a stick, the rotation can be reversed. The video shows the rotation being reversed twice. The reversal of the rotational direction corresponds to the reversal of magnetization in ferromagnetics. The angular velocity of the upper disk was approximately $9.5 \mathrm{mrad} / \mathrm{s}$. The time dependence of the shear rate is also depicted in Fig. 15.

\section{Measurements using a strain-controlled rheometer}

We also measured the shear-rate-shear-stress curve using a strain-controlled rheometer (ARES-G2, TA Instrument) [Fig. 16]. The diameter of the metal disk and the gap were identical to those in the main measurements using a stress-controlled rheometer (MCR-302, Anton Paar), although the measurement temperature was slightly higher $\left(27.1^{\circ} \mathrm{C}\right.$; cf. $25^{\circ} \mathrm{C}$ ). At $E=1.5 \mathrm{~V} / \mu \mathrm{m}$ and $f=50 \mathrm{~Hz}$, an S-shaped curve was clearly observed, although it was quantitatively slightly different from the corresponding curve shown in Fig. 4(b). This discrepancy may be attributable to the differences in the materials used for the upper disks and the lower stages, which were metal in the ARES-G2 system and ITO-coated glass in the MCR-302 system.
[1] J.-C. Bacri, R. Perzynski, M. I. Shliomis, and G. I. Burde, Phys. Rev. Lett. 75, 2128 (1995).

[2] H. M. López, J. Gachelin, C. Douarche, H. Auradou, and E. Clément, Phys. Rev. Lett. 115, 028301 (2015).

[3] A. Sokolov and I. S. Aranson, Phys. Rev. Lett. 103, 148101 (2009).

[4] S. Rafaï, L. Jibuti, and P. Peyla, Phys. Rev. Lett. 104, 098102 (2010).

[5] J. Gachelin, G. Miño, H. Berthet, A. Lindner, A. Rousselet, and E. Clément, Phys. Rev. Lett. 110, 268103 (2013).

[6] M. I. Shliomis and K. I. Morozov, Phys. Fluids 6, 2855 (1994).

[7] H. W. Müller and M. Liu, Phys. Rev. E 64, 061405 (2001).

[8] R. A. Simha and S. Ramaswamy, Phys. Rev. Lett. 89, 058101 (2002).

[9] Y. Hatwalne, S. Ramaswamy, M. Rao, and R. A. Simha, Phys. Rev. Lett. 92, 118101 (2004).

[10] B. M. Haines, A. Sokolov, I. S. Aranson, L. Berlyand, and D. A. Karpeev, Phys. Rev. E 80, 041922 (2009).

[11] L. Giomi, T. B. Liverpool, and M. C. Marchetti, Phys. Rev. E 81, 051908 (2010).

[12] D. Saintillan, Exp. Mech. 50, 1275 (2010).

[13] S. D. Ryan, B. M. Haines, L. Berlyand, F. Ziebert, and I. S. Aranson, Phys. Rev. E 83, 050904 (2011).

[14] A. Loisy, J. Eggers, and T. B. Liverpool, Phys. Rev. Lett. 121, 018001 (2018).

[15] T. Nagaya, M. Niu, S. Nara, Y. H. Na, and H. Orihara, Phys. Rev. E 87, 012501 (2013).

[16] T. Nagaya, Y. Satou, Y. Goto, Y. Hidaka, and H. Orihara, J. Phys. Soc. Jpn. 85, 074002 (2016).

[17] P. G. de Gennes and J. Prost, The Physics of Liquid Crystals, 2nd ed. (Oxford Science, New York, 1993).

[18] S. Chandrasekhar, Liquid Crystals, 2nd ed. (Cambridge University Press, Cambridge, 1992).

[19] H. Orihara, N. Sakurai, Y. Sasaki, and T. Nagaya, Phys. Rev. E 95, 042705 (2017).
[20] P. Sheng and W. Wen, Annu. Rev. Fluid Mech. 44, 143 (2012).

[21] K. Negita, Chem. Phys. Lett. 246, 353 (1995).

[22] K. Negita, J. Chem. Phys. 105, 7837 (1996).

[23] P. Patrício, C. Leal, L. Pinto, A. Boto, and M. Cidade, Liq. Cryst. 39, 25 (2012).

[24] G. H. Heilmeier, L. A. Zanoni, and L. A. Barton, Proc. IEEE 56, 1162 (1968).

[25] S. Kai, W. Zimmermann, M. Andoh, and N. Chizumi, Phys. Rev. Lett. 64, 1111 (1990).

[26] S. Nasuno, O. Sasaki, S. Kai, and W. Zimmermann, Phys. Rev. A 46, 4954 (1992).

[27] S. Kai, M. Andoh, and S. Yamaguchi, Phys. Rev. A 46, R7375 (1992).

[28] V. S. U. Fazio, F. Nannelli, and L. Komitov, Phys. Rev. E 63, 061712 (2001).

[29] T. Tóth-Katona and J. T. Gleeson, Phys. Rev. E 69, 016302 (2004).

[30] See Supplemental Material at http://link.aps.org/supplemental/ 10.1103/PhysRevE.99.012701 for videos.

[31] L. D. Landau and E. M. Lifshitz, Statistical Physics, 3rd ed. (Pergamon Press, Oxford, 1980).

[32] D. K. Roy, Tunnelling and Negative Resistance Phenomena in Semiconductors (Pergamon Press, Oxford, 1977).

[33] J. W. Strutt, Scientific Papers (Macmillan, New York, 1943).

[34] B. van der Pol Jun, Philos. Mag. 2, 978 (1926).

[35] C. Gähwiller, Mol. Cryst. Liq. Cryst. 20, 301 (1973).

[36] D. Diguet, F. Rondelez, and G. Durand, C. R. Acad. Sci. B 271, 954 (1970).

[37] G. Sivashinsky and V. Yakhot, Phys. Fluids 28, 1040 (1985).

[38] G. I. Sivashinsky and A. L. Frenkel, Phys. Fluids A 4, 1608 (1992).

[39] C. Meneveau and J. Katz, Annu. Rev. Fluid Mech. 32, 1 (2000).

[40] K. Tomita and H. Tomita, Prog. Theor. Phys. 51, 1731 (1974). 